\title{
Functional characterization of a mammalian transcription factor, Elongin A
}

Takashi Yasukawa, ${ }^{1}$ Kazuto Sugimura, ${ }^{2}$ Mizue Fukuda $^{3}$ Katsuhisa Yamazaki, Shigetaka Kitajima, ${ }^{3}$ Katsuzumi Okumura ${ }^{2}$ and Teijiro Aso ${ }^{*}, 1$

From the ${ }^{1}$ Department of Functional Genomics, Kochi Medical School, Kohasu, Oko-cho, Nankoku, Kochi 783-8505, Japan; ${ }^{2}$ Laboratory of Molecular and Cellular Biology, Faculty of Bioresources, Mie University, 1515 Kamihama, Tsu, Mie 514-8507, Japan; ${ }^{3}$ Department of Biochemical Genetics, Medical Research Institute, Tokyo Medical and Dental University, Yushima, Bunkyo-ku, Tokyo 113-8510, Japan; ${ }^{4}$ Microbial Chemistry Research Foundation, Kamiosaki, Shinagawa-ku, Tokyo 141-0021, Japan

*Corresponding author: Teijiro Aso, Department of Functional Genomics, Kochi Medical School, Kohasu, Oko-cho, Nankoku, Kochi 783-8505, Japan

TEL: +81-88-880-2279; Fax: +81-88-880-2281; E-mail: asot@med.kochi-u.ac.jp 


\begin{abstract}
Elongin $\mathrm{A}$ is the transcriptionally active subunit of the Elongin complex that strongly stimulates the rate of elongation by RNA polymerase II (pol II) by suppressing the transient pausing of the polymerase at many sites along the DNA template. We have recently shown that Elongin A-deficient mice are embryonic lethal, and mouse embryonic fibroblasts (MEFs) derived from Elongin $\mathrm{A}^{-/-}$embryos display not only increased apoptosis but also senescence-like phenotypes accompanied by the activation of p53. To further understand the function of Elongin A in vivo, we have carried out the structure-function analysis of Elongin A and identified sequences critical to its nuclear localization and direct interaction with pol II. Moreover, we have analyzed the replication fork movement in wild-type and Elongin $\mathrm{A}^{-/-} \mathrm{MEFs}$, and shown the possibility that the genomic instability observed in Elongin $\mathrm{A}^{-/-}$MEFs might be caused by the replication fork collapse due to Elongin A deficiency.
\end{abstract}

Keywords: Elongin A; transcription elongation; RNA polymerase II; replication; genomic instability 
Eukaryotic messenger RNA synthes is by RNA polymerase II (pol II) is regulated by the concerted action of a set of general transcription factors that control the activity of pol II during the initiation and elongation stages of transcription. At least six general transcription initiation factors (TFIIA, TFIIB, TFIID, TFIIE, TFIIF and TFIIH) have been identified in eukaryotic cells and found to promote the selective binding of pol II to promoters and to support a basal level of transcription [1]. In addition, a diverse collection of elongation factors that promote the efficient elongation of transcripts by pol II on naked DNA templates in vitro have been identified biochemically [2-4]. These elongation factors fall into two broad functional classes based on their ability to either reactivate arrested pol II or suppress the transient pausing of pol II. The first class is composed of members of the SII family $[2,5]$. The second class is comprised of a collection of elongation factors, including TFIIF [6], Elongin [7, 8], ELL [9] and Cockayne syndrome B (CSB) protein [10], which increase the overall rate of mRNA chain elongation by decreasing the frequency and/or duration of the transient pausing of pol II at sites along the DNA template.

Elongin was initially identified as a heterotrimeric protein composed of A, B and C subunits of $\sim 770,118$ and 112 amino acids, respectively $[7,8,11,12]$. Elongin $A$ is the transcriptionally active subunit, whereas Elongins $\mathrm{B}$ and $\mathrm{C}$ are positive regulatory subunits that can form an isolable Elongin $\mathrm{BC}$ subcomplex $[8,13,14]$. Recently, we have generated Elongin A-deficient mice by gene targeting [15]. Elongin $\mathrm{A}^{-/-}$embryos exhibited severely retarded growth and died at between days 10.5 and 12.5 of gestation, most likely due to excessive apoptosis. Moreover, mouse embryonic fibroblasts (MEFs) derived from Elongin $\mathrm{A}^{-/}$embryos displayed not only increased apoptosis but also senescence-like growth defects accompanied by the activation of p53.

As part of our effort to understand the function and regulation of Elongin A in vivo, we have carried out the structure-function analysis of Elongin A and identified sequences critical for its nuclear localization and direct interaction with pol II. In addition, we have analyzed the rate of replication fork movement in wild-type and Elongin $\mathrm{A}^{-/-}$MEFs, and demonstrated the possibility that the genomic instability observed in Elongin $\mathrm{A}^{-/}$MEFs might be caused by the replication fork collapse due to the lack of Elongin A. 


\section{Materials and methods}

Plasmid construction. DNA constructs expressing Elongin A mutants were generated by oligonucleotide-directed mutagenes is of pcDNA3.1-FLAG-Elongin A [16] using the QuickChange site-directed mutagenesis kit (Stratagene). For the expression of glutathione S-transferase (GST)-Elongin A fusion protein, cDNA encoding the entire rat Elongin A was amplified by PCR from pSVL-Elongin A [13], and subcloned into the BamHI and SalI sites of pGEX-4T1 (Amersham). Subsequently, constructs expressing GST fused to various Elongin A mutants were generated by oligonucleotide-directed mutagenesis using the QuickChange site-directed mutagenesis kit (Stratagene).

Expression of recombinant proteins in Escherichia coli and in vitro binding assay. GST and GST-Elongin A fusion proteins were expressed in E. coli DH5 $\alpha$ and purified using glutathione-sepharose affinity chromatography (Amersham) as described [16] according to the manufacturer's instructions. pol II was purified as described from rat liver nuclear extracts [17]. Immobilized GST fusion proteins $(\sim 15 \mu \mathrm{g})$ were mixed with purified pol II and incubated for $1 \mathrm{~h}$ at $4^{\circ} \mathrm{C}$. The beads were then washed five times with buffer containing $20 \mathrm{mM}$ Hepes- $\mathrm{NaOH}$ (pH 7.9), $500 \mathrm{mM} \mathrm{NaCl}, 2.5 \mathrm{mM} \mathrm{MgCl} 2,50$ $\mathrm{mM} \mathrm{ZnCl} 2,0.1 \mathrm{mM}$ EDTA, $0.05 \%$ Nonidet P-40 and $0.5 \mathrm{mM}$ DTT. Aliquots of loaded and bound fractions were subjected to SDS-polyacrylamide gel electrophoresis (PAGE), transferred to a polyvinylidene difluoride membrane (Millipore), and probed with antipol II (8WG16) antibody (Babco). Horseradish peroxidase-conjugated sheep anti-mouse antibody (Amersham) was used as a secondary antibody. Detection was performed using SuperSignal West Dura Extended Duration Substrate (Pierce).

Immunofluorescence staining. Immunostaining of cells was performed as previously described [16, 18]. Briefly, COS 7 cells were cultured in Dulbecco's modified Eagle's medium (DMEM) supplemented with 10\% fetal bovine serum (FBS) and were transiently transfected with the pcDNA3.1/Hygro-FLAG-wild-type or mutant Elongin A using FuGENE 6 (Roche) accord ing to the manufacturer's protocol. The cells grown in a chamber slide were fixed by immersion in cold acetone/methanol (1:1) for $10 \mathrm{~min}$, then rinsed with $70 \%$ ethanol, $50 \%$ ethanol and finally phosphate-buffered saline (PBS). After blocking in PBS containing 2\% bovine serum albumin, $0.2 \%$ Tween 20 and $6.7 \%$ 
glycerol at $4{ }^{\circ} \mathrm{C}$ overnight, the cells were incubated for $1 \mathrm{~h}$ at $4{ }^{\circ} \mathrm{C}$ with rabbit polyclonal anti-FLAG antibody (1:1000) and mouse monoclonal anti-HA antibody. Reacting antibodies were stained with fluorescein-conjugated goat anti-rabbit IgG and rhodamine-conjugated goat anti-mouse $\operatorname{IgG}_{2 b}$ (Santa Cruz). Fluorescence microscopy of fixed cells was performed using an Olympus BX-50 confocal laser scanning microscope.

Generation of Elongin A-deficient mice. Elongin A-deficient mice were generated as previously described [15]. Briefly, Elongin A mutant ES cell clones were microinjected in to blastocysts for the generation of chimeric mice. Chimeric males were crossed with C57BL/6 females, and agouti-colored offspring were analyzed for the germline transmission of the Elongin A mutation. Heterozygous animals were intercrossed to generate homozygous mutant animals.

PCR genotyping. Genomic DNA was isolated from embryonic yolk sacs, and genotyping was performed using PCR as described [15].

Preparation of MEFs. MEFs were prepared from E10.5 embryos as described [15]. Briefly, fetal tissue samples were rinsed in PBS and then were mechan ically dissociated in DMEM with 10\% FBS. The cells were then plated out on 6-well plates. These cells were considered to be passage 1 MEFs.

Quantitative analysis of apoptosis in MEFs. For the quantitative analysis and to determine the cytoplasmic histone-associated DNA fragments, which are indicative of on-going apoptosis, the Cell Death Detection ELISA ${ }^{\text {PLUS }}$ (Roche Applied Science) was used according to the manufacturer's instructions.

Replication labeling. Cells were labeled with biotin-16-dUTP and digoxigenin-11-dUTP (Roche Applied Science) at 20-min or 30-min intervals using hypotonic shift procedure with slight modifications [19]. Cells grown on a $35-\mathrm{mm}$-diameter dish were washed with $1 \mathrm{ml}$ of $\mathrm{KH}$ buffer $(30 \mathrm{mM} \mathrm{KCl}, 10 \mathrm{mM}$ HEPES, pH 7.4) and then $10 \mu \mathrm{l}$ of $\mathrm{KH}$ buffer supplemented with $250 \mu \mathrm{M}$ biotin-16-dUTP or digoxigenin-11-dUTP (Roche Applied Science) was added. After incubation at $37^{\circ} \mathrm{C}$ with $5 \% \mathrm{CO}_{2}$ for $10 \mathrm{~min}$, the samples were washed with serum-free medium and cultured in normal medium for $20 \mathrm{~min}$ or $30 \mathrm{~min}$ to introduce modified nucleotides into nascent DNA. DNA fiber preparation was performed as described 
previously [20, 21]. Replication labeled cells and 10- to 20-fold unlabeled cells were mixed before sample preparation because DNA fibers were extended straight and each replication-labeled DNA fiber was well separated. Cells were fixed with methanol/acetic acid (3:1) solution, dropped onto a slideglass, and then dipped into lysis buffer $\left(0.5 \%\right.$ SDS, $200 \mathrm{mM}$ Tris-HCl, $\mathrm{pH} 7.4,50 \mathrm{mM}$ EDTA) for $10 \mathrm{~min}$ at $25^{\circ} \mathrm{C}$. DNA fibers were allowed to drop down from nuclei, dried and fixed with methanol/acetic acid (3:1) solution for $3 \mathrm{~min}$ at $25^{\circ} \mathrm{C}$. Biotin- and digoxigenin-labeled DNAs were detected using Alexa488-streptavidin (Invitrogen) and anti-digoxigenin conjugated with rhodamine (Roche Applied Science), respectively.

Microarray analysis. Total RNA was isolated from Elongin $\mathrm{A}^{+/+}$and Elongin $\mathrm{A}^{-/-}$ MEFs according to the manufacturer's protocol using an RNeasy Mini kit (Qiagen). One-microgram aliquots of total RNA were labeled using the Agilent Linear Amplification/Labeling kit (Agilent Technologies) according to the manufacturer's instructions. After checking the labeling efficiency, 1- $\mu \mathrm{g}$ aliquots of Cy3-labeled Elongin $\mathrm{A}^{+/+}$cRNA and Cy5-labeled Elongin $\mathrm{A}^{-/}$cRNA were mixed, then hybridized to an Agilent Whole Mouse Oligo Microarray using the manufacturer's hybridization protocol. After the washing step, the microarray slide was analyzed with an Agilent Microarray scanner and software (scanner model G2505B; software G2565BA). A data analysis was performed using the Agilent Feature Extraction software (Version A.6.1.1).

\section{Results and discussion}

\section{Identification of the nuclear localization signals of Elongin A}

We have previously shown that Elongin A and its related family proteins are predominantly localized in the cell nucleus $[16,18]$. In this study, we analyzed the sequences of Elongin A required for its nuclear localization using immunostaining assays. COS7 cells were transfected with constructs expressing wild-type or various deletion mutants of Elongin A with FLAG-tag at their $\mathrm{NH}_{2}$-terminus, and stained with anti-FLAG antibody. As shown in Fig. 1, the Elongin A mutant composed of residues 400-589 was clearly localized in the nucleus. Analysis of the amino acid sequences in 
this region of Elongin A indicated four short sequences rich in basic amino acids that resemble the potential nuclear localization signals (NLSs): amino acids 413 to 418 (NLS1; RKKKKK), 433 to 435 (NLS2; KKK), 470 to 473 (NLS3; RPRK), and 509 to 511 (NLS4; KRK) [22, 23]. In order to determine whether any of these sequences is functional, four additional deletion mutants that lacked either NLS1, NLS2, NLS3 or NLS4 from residues 400-589 of Elongin A were constructed. Transfection of COS7 cells with expression vectors containing these deletion mutants demonstrated that NLS1, but not NLS2, NLS3 or NLS4, was required for nuclear localization, as the encoded protein missing NLS1 was identified in the cytoplasm (Fig. 1B). In contrast, the deletion of any of NLS2, NLS3 or NLS4 did not disrupt the nuclear localization of the encoded protein.

Identification of Elongin A region responsible for the direct interaction with pol II

In previous studies, we have demonstrated that Elongin $\mathrm{A}$ is capable of stimulating the rate of transcription elongation in vitro in the reaction mix containing only DNA template and pol II, and that the $\mathrm{COOH}$ terminus of Elongin A (residues 400-773) possesses a level of elongation stimulatory activity comparable to full-length Elongin A $[8,14]$. From these findings, it was postulated that Elongin A interacts with pol II in a direct manner and that the amino acid sequences responsible for this interaction reside in residues 400-773 of Elongin A.

Thus, to confirm the above presumptions, various truncation mutants of Elongin A were fused to GST, expressed in E. coli, and immobilized on glutathione-sepharose resin. These resins were then incubated with purified pol II. After extensive washing, the bound protein was eluted, fractionated on an SDS-PAGE gel, and probed with 8WG16 antibody. As shown in Fig. 2, pol II was retained by the GST-Elongin A(400-773) affinity resins, while no detectable pol II was retained by the resin containing GST-Elongin A(1-399). Subsequent analysis demonstrated that GST fused with residues 590-690 of Elongin A bound to pol II. Meanwhile, GST fused with residues 1-120 of Elongin $A$, which is $29 \%$ identical and $53 \%$ similar to the $\mathrm{NH}_{2}$-terminus of SII and has been shown to interact with pol II holoenzyme complex [24], failed to bind to purified pol II. 
Taken together, these results suggest: (i) residues 590-690 of Elongin A, which is the region highly conserved between Elongin A and its related family members, Elongins A2 and A3 [18], are responsible for the direct interaction with pol II; and (ii) interaction of the $\mathrm{NH}_{2}$ terminus of Elongin A with pol II seems to be indirect, and most likely mediated through some of the components of the pol II holoenzyme complex.

\section{Induction of genomic instability in Elong in $A^{-/-} M E F S$}

To gain more insight into the role of Elongin A in vivo, we have recently generated Elongin A-deficient mice by gene targeting [15]. As shown in Fig. 3A, MEFs prepared from Elongin $\mathrm{A}^{-/}$embryos indeed lack the endogenous Elongin A protein. As Elongin $\mathrm{A}^{-/-}$MEFs at E10.5 exhibited a slower growth rate compared with wild-type and Elongin $\mathrm{A}^{+/-}$MEFs, we have quantitatively analyzed the apop to tic status of E10.5 MEFs using cell death detection ELISA assays. Consistent with the findings obtained by annexin V-FITC and propidium iodide (PI) staining [15], Elongin $\mathrm{A}^{-/-}$MEFs showed a significant increase in histone-associated DNA fragmentation compared with the wild-type MEFs (Fig. 3B). Moreover, Elongin $\mathrm{A}^{-/-}$MEFs displayed the features of premature senescence, including a flattened and enlarged morphology and an elevated activity of senescence-associated $\beta$-galactosidase, accompanied by the activation of p53 [15], suggesting that Elongin $\mathrm{A}^{-/-}$MEFs had sustained genomic instability due to the lack of Elongin A.

Inhibition of the elongation phase of transcription by various agents, such as UV light, camptothecin or actinomycin $\mathrm{D}$, has also been reported to lead to the activation of p53 and the induction of apoptosis $[25,26]$. The induction of apoptosis following elongation blockage has been shown to occur preferentially in the $\mathrm{S}$ phase of the cell cycle [27]. As transcription also persists during the $\mathrm{S}$ phase of the cell cycle [28], it is possible that oncoming replication forks might occasionally collide with the blocked pol II elongation complexes, and that these collisions might lead to the stalling and breakdown of replication forks, resulting in genomic instability and the triggering of apoptosis.

The rate of replication fork movement is significan tly reduced in Elong in $A^{-/-} M E F s$ 
Thus, as an initial step to examine this possibility, we measured the rates of replication fork movement in non-synchronized wild-type and Elongin $\mathrm{A}^{-/-}$MEFs using the hypotonic shift method. The histograms in Fig. 4A represent the distribution of distances, where each replication fork has moved on DNA fibers during either $20 \mathrm{~min}$ (left panels) or $30 \mathrm{~min}$ (right panels). A fairly scattered pattern of distribution was observed. This is probably reflects the fact that the rate of replication fork movement is not constant during the $\mathrm{S}$ phase of the cell cycle [21]. Most notably, however, the proportion of the replication forks that had progressed only a short distance, i.e., less than $20 \mathrm{~kb}$, within a given time was significantly larger in Elongin $\mathrm{A}^{-/-}$MEFs than in wild-type MEFs. The average rate of replication fork movement in Elongin $\mathrm{A}^{-/-} \mathrm{MEFs}$ was calculated to be approximately two-thirds of that in wild-type MEFs (Fig. 4B). These findings, therefore, support the possibility that the genomic instability observed in cells with transcriptional elongation impairment, including Elongin $\mathrm{A}^{-/-} \mathrm{MEF}$ s, could arise as a consequence of the replication fork collapse after collision with the blocked pol II (Fig. 4C).

Next, to rule out the possibility that the observed reduction of the replication fork movement in Elongin $\mathrm{A}^{-/-}$MEFs is caused by the decreased expression of the factors involved in DNA replication at the transcriptional level by Elongin A deficiency, a cDNA microarray analysis comparing wild-type MEFs with Elongin $\mathrm{A}^{-/-}$MEFs was performed. Under our experimental cond itions, mRNAs whose levels were decreased or increased by 2 -fold or greater were considered to be differentially expressed transcripts. As shown in Table 1, the expression of the genes whose translational products play important roles in regulating the process of DNA replication was either not or was only weakly altered in Elongin $\mathrm{A}^{-/-}$MEFs, suggesting that the deficiency of replication factors might not be the cause of the replication fork impairment in Elongin $\mathrm{A}^{-/-}$MEFs. In addition, the expression levels of the pol II elongation factor-related genes, excluding Elongin A, did not significantly decrease.

In summary, we have carried out the structure-function analysis of transcription elongation factor Elongin A and identified sequences critical for its function-that is, i) nuclear localization, and ii) interaction with pol II. In addition, we have analyzed the rate of replication fork movement in wild-type and Elongin $\mathrm{A}^{-/-}$MEFs, and shown the 
possibility that the genomic instability observed in Elongin $\mathrm{A}^{-/-}$MEFs might be caused by the replication fork collapse due to the lack of Elongin A. Therefore, it seems reasonable to speculate that Elongin A in cells plays roles not only in quantitatively regulating the synthesis of mRNA but also in maintaining the genomic stability by preventing pausing or arrest of the transcribing pol II.

\section{Acknowledgments}

We would like to thank A. Tsutsui and K. Tamura for their technical assistance. This work was supported by grants from the Ministry of Education, Culture, Sports, Science and Technology of Japan; the Yamanouchi Foundation for Research on Metabolic Disorders; and the Fugaku Trust for Medical Research.

\section{References}

[1] R.G. Roeder, The role of general initiation factors in transcription by RNA polymerase II, Trends Biochem. Sci. 21 (1996) 327-335.

[2] J.W. Conaway, A. Shilatifard, A. Dvir, R.C. Conaway, Control of elongation by RNA polymerase II, Trends Biochem. Sci. 25 (2000) 375-380.

[3] A. Shilatifard, R.C. Conaway, J.W. Conaway, The RNA polymerase II elongation complex, Annu. Rev. Biochem. 72 (2003) 693-715.

[4] R.J. Sims III, R. Belotserkovskaya, D. Reinberg, Elongation by RNA polymerase II: the short and long of it, Genes Dev. 18 (2004) 2437-2468.

[5] M. Wind, D. Reines, Transcription elongation factor SII, BioEssays 22 (2000) 327-336.

[6] D.H. Price, A.E. Sluder, A.L. Greenleaf, Dynamic interaction between a Drosophila transcription factor and RNA polymerase II, Mol. Cell. Biol. 9 (1989) 1465-1475.

[7] J.N. Bradsher, K.W. Jackson, R.C. Conaway, J.W. Conaway, RNA polymerase II transcription factor SIII. I. Identification, purification, and properties, J. Biol. Chem. 268 (1993) 25587-25593.

[8] T. Aso, W.S. Lane, J.W. Conaway, R.C. Conaway, Elongin (SIII): A multisubunit regulator of elongation by RNA polymerase II, Science 269 (1995) 
1439-1443.

[9] A. Shilatifard, W.S. Lane, K.W. Jackson, R.C. Conaway, J.W. Conaway, An RNA polymerase II elongation factor encoded by the human ELL gene, Science 271 (1996) 1873-1876.

[10] C.P. Selby, A. Sancar, Cockayne syndrome group B protein enhances elongation by RNA polymerase II, Proc. Natl. Acad. Sci. USA 94 (1997) 11205-11209.

[11] K.P. Garrett, S. Tan, J.N. Bradsher, W.S. Lane, J.W. Conaway, R.C. Conaway, Molecular cloning of an essential subunit of RNA polymerase II elongation factor SIII, Proc. Natl. Acad. Sci. USA 91 (1994) 5237-5241.

[12] K.P. Garrett, T. Aso, J.N. Bradsher, S.I. Foundling, W.S. Lane, R.C. Conaway, J.W. Conaway, Positive regulation of general transcription factor SIII by a tailed ubiquitin homolog, Proc. Natl. Acad. Sci. USA 92 (1995) 7172-7176.

[13] D.R. Duan, A. Pause, W.H. Burgess, T. Aso, D.Y.T. Chen, K.P. Garrett, R.C. Conaway, J.W. Conaway, W.M. Linehan, R.D. Klausner, Inhibition of transcription elongation by the VHL tumor suppressor protein, Science 269 (1995) 1402-1406.

[14] T. Aso, D. Haque, R.J. Barstead, R.C. Conaway, J.W. Conaway, The inducible elongin A elongation activation domain: structure, function and interaction with the elongin BC complex, EMBO J. 15 (1996) 5557-5566.

[15] K. Miyata, T. Yasukawa, M. Fukuda, T. Takeuchi, K. Yamazaki, K. Sakumi, M. Tamamori-Adachi, Y. Ohnishi, Y. Ohtsuki, Y. Nakabeppu, S. Kitajima, S. Onishi, T. Aso, Induction of apoptosis and cellular senescence in mice lacking transcription elongation factor, Elongin A, Cell Death Differ., in press.

[16] K. Tamura, K. Miyata, K. Sugahara, S. Onishi, T. Shuin, T. Aso, Identification of EloA-BP1, a novel Elongin A binding protein with an exonuclease homology domain, Biochem. Biophys. Res. Commun. 309 (2003) 189-195.

[17] J.W. Conaway, R.C. Conaway, An RNA polymerase II transcription factor shares functional properties with Escherichia coli $\sigma 70$, Science 248 (1990) 1550-1553.

[18] K. Yamazaki, L. Guo, K. Sugahara, C. Zhang, H. Enzan, Y. Nakabeppu, S. Kitajima, T. Aso, Identification and biochemical characterization of a novel transcription elongation factor, Elongin A3, J. Biol. Chem. 277 (2002) 26444-26451. 
[19] Koberna, K., D. Stanek, J. Malinsky, M. Eltsov, A. Pliss, V. Ctrnacta, S. Cermanova, and I. Raska, Nuclear organization studied with the help of a hypotonic shift: its use permits hydrophilic molecules to enter into living cells, Chromosoma 108 (1999) 325-335.

[20] S. Takebayashi, E.M.M. Manders, H. Kimura, H. Taguchi, K. Okumura, Mapping sites where replication initiates in mammalian cells using DNA fibers, Exp. Cell Res. 271 (2001) 263-268.

[21] S. Takebayashi, K. Sugimura, T. Saito, C. Sato, Y. Fukushima, H. Taguchi, K. Okumura, Regulation of replication at the $\mathrm{R} / \mathrm{G}$ chromosomal band boundary and pericentromeric heterochromatin of mammalian cells, Exp. Cell Res. 304 (2005) 162-174.

[22] T. Boulikas, Nuclear localization signals (NLS), Crit. Rev. Eukar. Gene 3 (1993) 193-227.

[23] I.W. Mattaj, L. Englmeier, Nucleocytoplasmic transport: the soluble phase, Annu. Rev. Biochem. 67 (1998) 265-306.

[24] G. Pan, T. Aso, J. Greenblatt, Interaction of elongation factors TFIIS and Elongin A with a human RNA polymerase II holoenzyme capable of promoter-specific initiation and responsive to transcriptional activators, J. Biol. Chem. 272 (1997) 24563-24571.

[25] M. Ljungman, F. Zhang, Blockage of RNA polymerase as a possible trigger for u.v. light-induced apoptosis, Oncogene 13 (1996) 823-831.

[26] M. Ljungman, H.M. O’Hagan, M.T. Paulsen, Induction of ser15 and lys382 modifications of p53 by blockage of transcription elongation, Oncogene 20 (2001) 5964-5971.

[27] B.C. McKay, C. Becerril, J.C. Spronck, M. Ljungman, Ultraviolet light-induced apoptosis is associated with S-phase in primary human fibroblasts, DNA Repair 1 (2002) 811-820.

[28] P. McGlynn, R.G. Lloyd, Recombinational repair and restart of damaged replication forks, Nature Rev. Mol. Cell. Biol. 3 (2002) 859-870. 


\section{Figure legends}

Fig. 1. Analysis of Elongin A region required for nuclear localization. (A) Structures of Elongin A mutants used in this study. On the right, the results of the assays shown in (B) are shown. (B) The subcellular localization of wild-type and mutant Elongin A. COS7 cells were transfected with FLAG-tagged wild-type or mutant Elongin A. After $12 \mathrm{~h}$, the cells were stained for wild-type or mutant Elongin A using anti-FLAG antibody (green).

Fig. 2. Analysis of Elongin A region required for binding to pol II. (A) Structures of Elongin A mutants used to produce chimeras with GST protein. On the right, the results of the assays shown in (B) are shown. (B) Purified pol II was incubated with GST (lanes 2 and 10) or GST-Elongin A mutants (lanes 3-8 and 11-14). After extensive washing, the bound fractions were subjected to SDS-PAGE followed by Western blotting using 8WG16 antibody. Ten percent of the total input of pol II was loaded in lanes 1 and 9.

Fig. 3. Increased apoptosis in Elongin $\mathrm{A}^{-/-}$MEFs. (A) An expression analysis of endogenous Elongin A protein. Elongin $\mathrm{A}^{+/+}$, Elongin $\mathrm{A}^{+/-}$, and Elongin $\mathrm{A}^{-/-}$MEFs at passage 2 were analyzed by Westem blotting using rabbit antiserum raised against the nonconserved amino-acid sequences (residues 758-772) located in the $\mathrm{COOH}$-terminal portion of human Elongin A; $\alpha$-tubulin was used as a loading control. (B) A quantitative analysis of apoptosis in Elongin $\mathrm{A}^{+/+}$and Elongin $\mathrm{A}^{-/-}$MEFs. The level of apoptosis in MEFs was determined by ELISA quantification of cytoplasmic histone-associated DNA fragments.

Fig. 4. Reduction of the rate of replication fork movement in Elongin $\mathrm{A}^{-/-}$MEFs. (A) Distribution of the distances that replication forks moved either in $20 \mathrm{~min}$ (left panels) or 30 min (right panels) in Elongin $\mathrm{A}^{+/+}$(top) and Elongin $\mathrm{A}^{-/-}$(bottom) MEFs. (B) Rates of replication fork movement in Elongin $\mathrm{A}^{+/+}$and Elongin $\mathrm{A}^{-/-}$MEFs. (C) $\mathrm{A}$ model to explain how the Elongin A deficiency causes genomic instability. The stalled pol II elongation complex may block the progression of replication fork movement. This would lead to a replication fork collapse, which could subsequently generate 
recombinogenic DNA breaks or replication fork reversal. hnRNA, heterogeneous nuclear RNA. 
Click here to download high resolution image

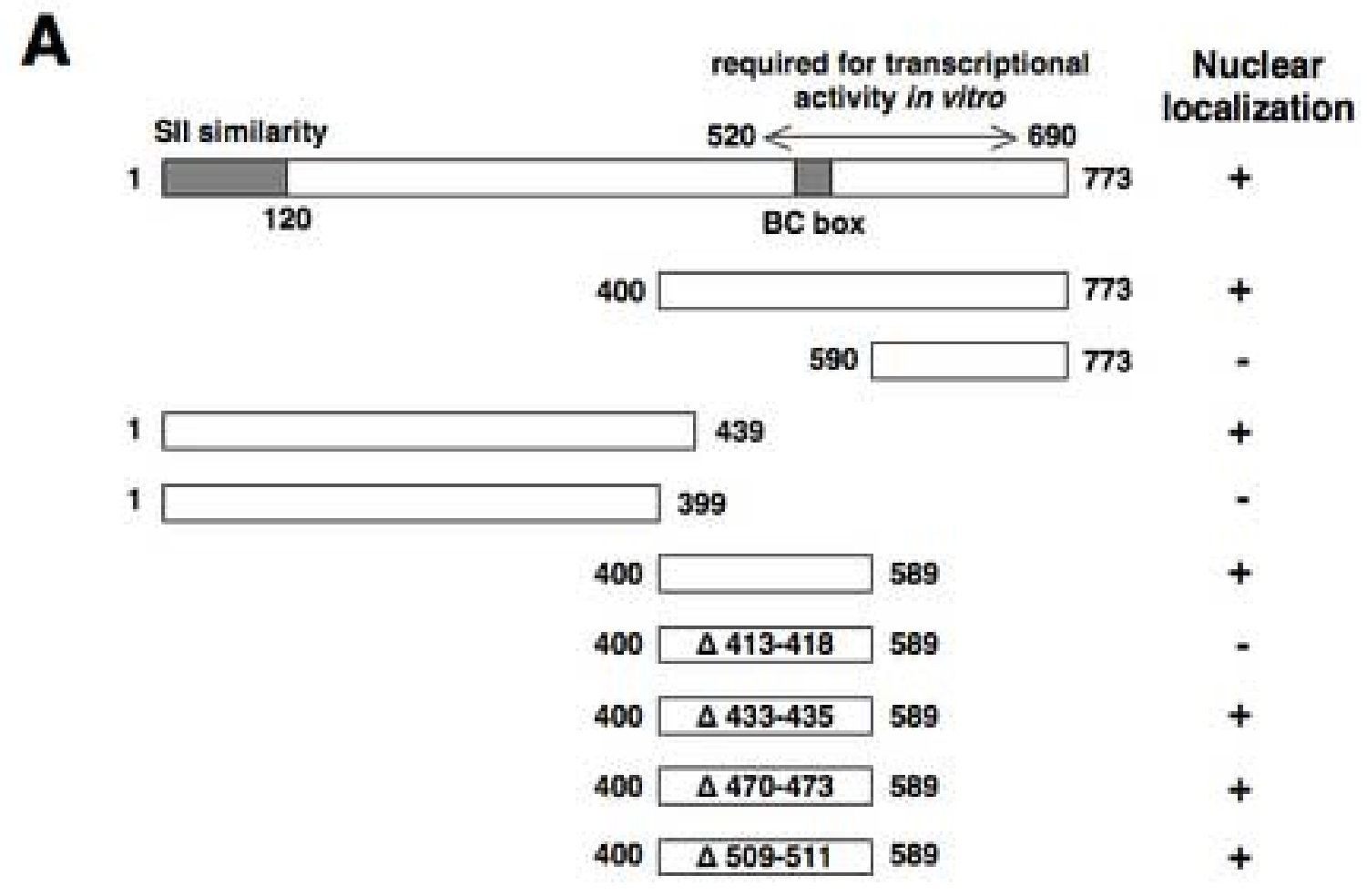

B
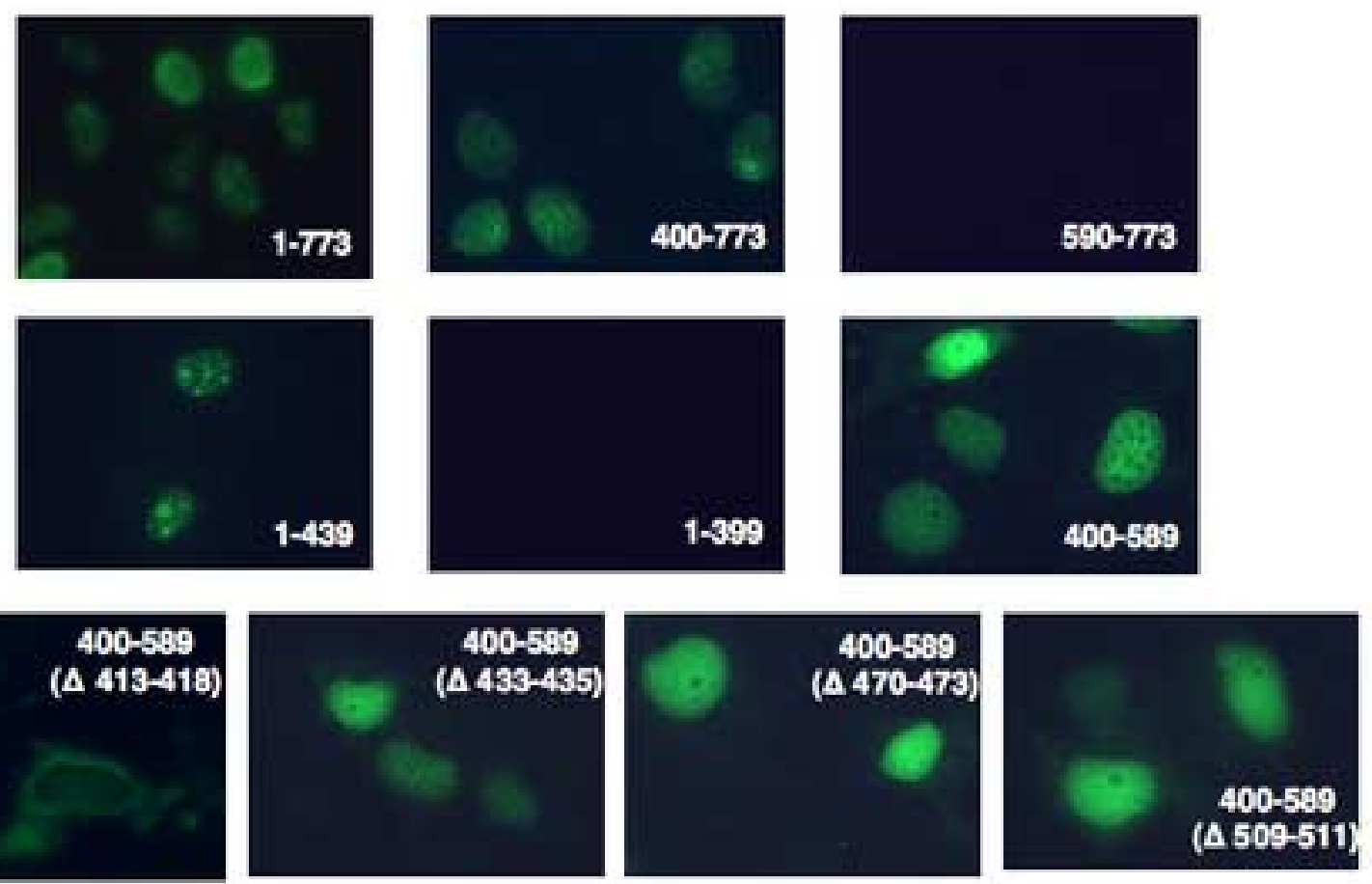

Figure 1 
Figure 2

Click here to download high resolution image

A

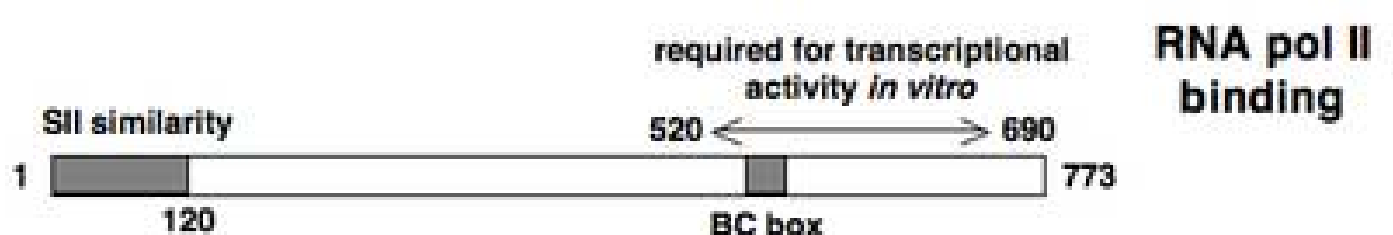

1

399

$1 \square 120$
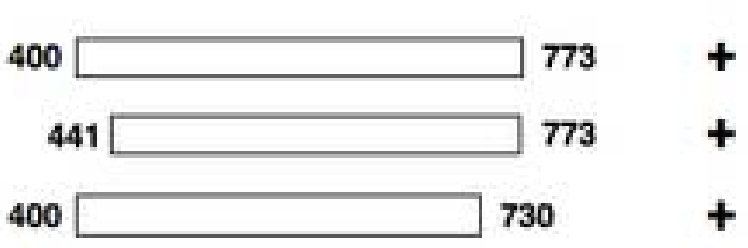

400

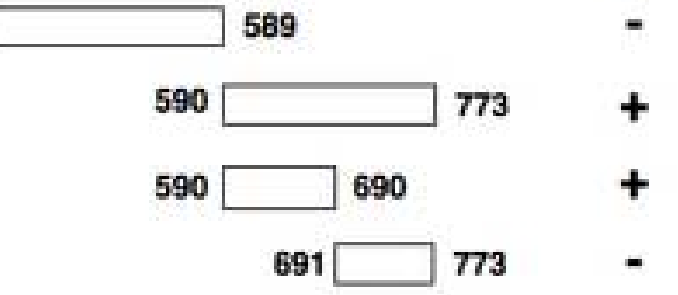

B
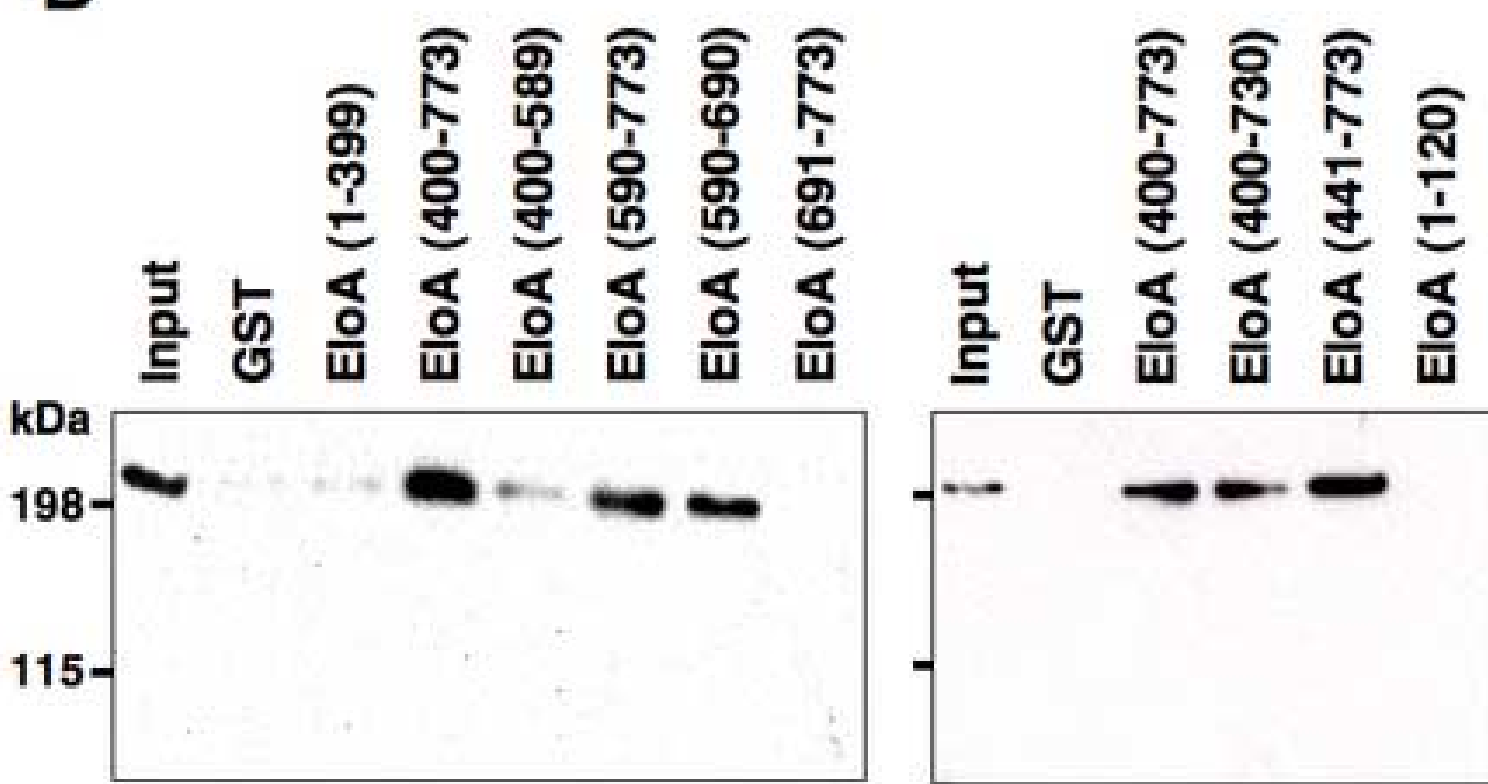

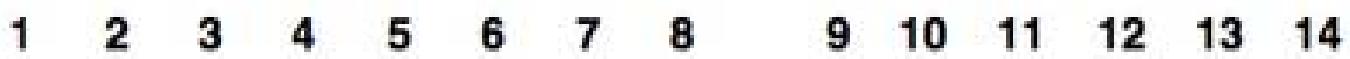

Figure 2 
Click here to download high resolution image

A

$+/++/++/-+/-\quad-/-\quad-/-$

\section{Elongin A}

\section{$\alpha$-Tubulin}

B

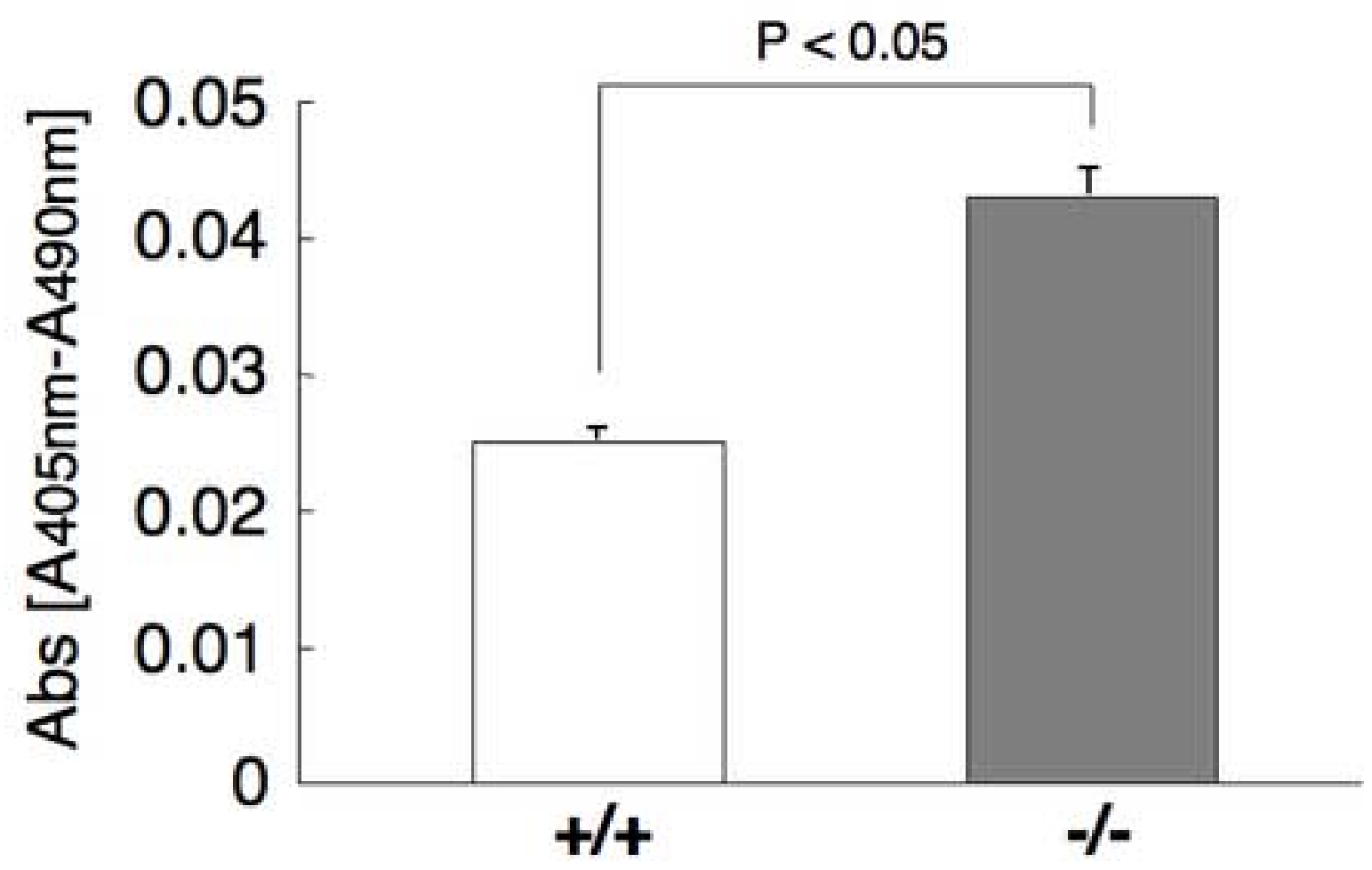

Figure 3 
Click here to download high resolution image

A
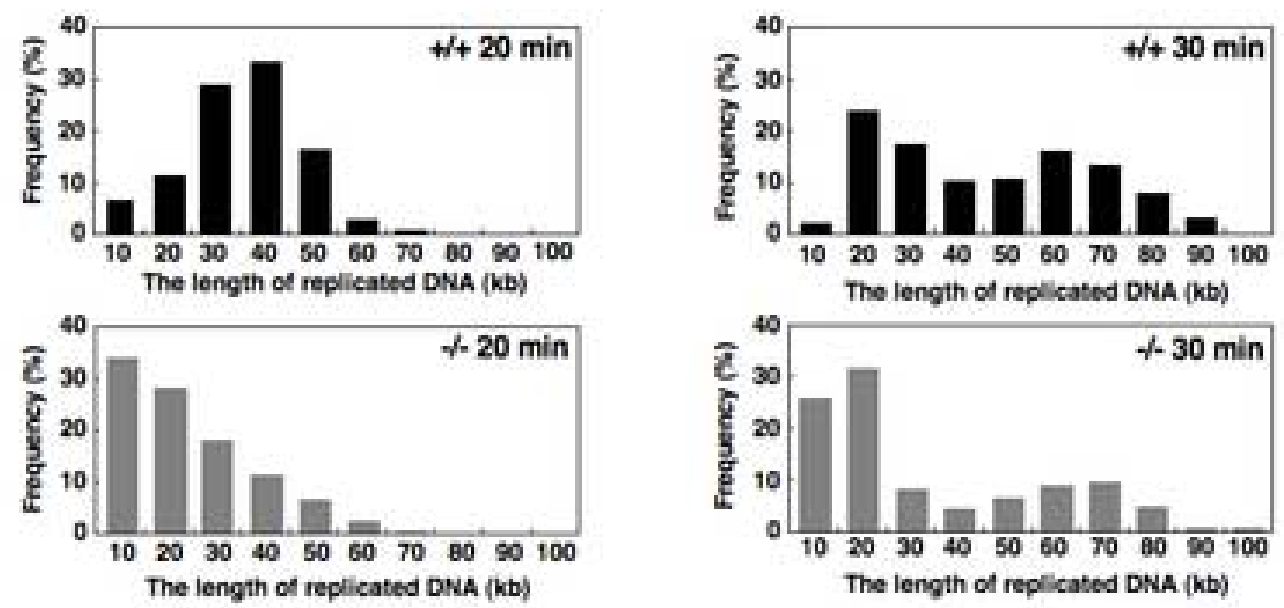

B

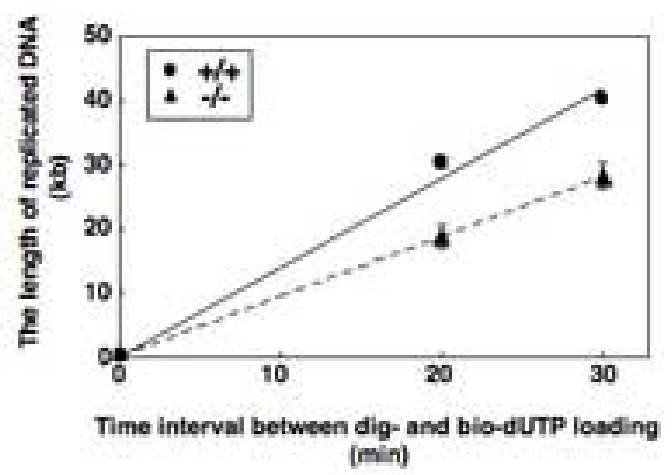

C

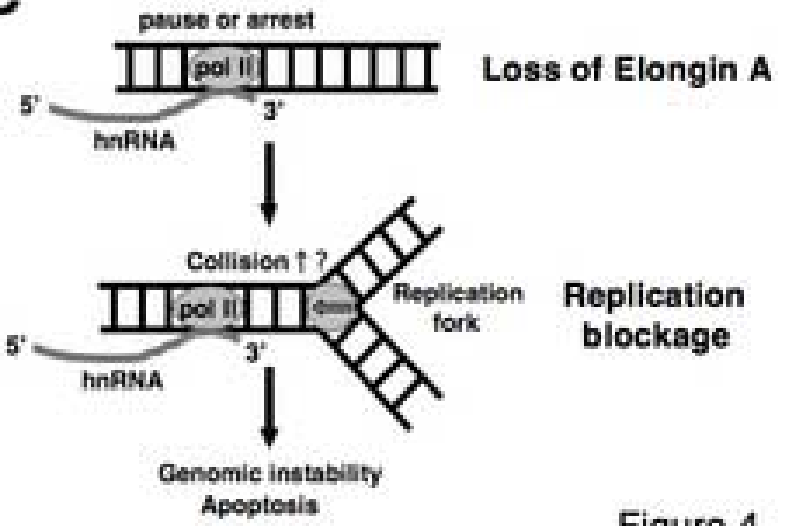


Table 1 Changes in the expression of factors required for DNA replication and transcription elongation in wild-type and Elongin $A^{-/-}$MEFs.

\section{A. DNA replication}

Gene Name

DNA polymerase $\alpha 1,180 \mathrm{kD}$ subunit (Pola 1 )

DNA polymerase $\alpha 2,68 \mathrm{kD}$ subunit (Pola2)

DNA polymerase $\beta$ (Polb)

DNA polymerase $\delta 1$, catalytic domain (Pold1)

DNA polymerase $\varepsilon$ (Pole)

Proliferating cell nuclear antigen (Pcna)

Replication protein $\mathrm{A}, 30 \mathrm{kD}$ subunit

Replication factor C1 (Recc1)

Topoisomerase II $\alpha$ (Top2a)

DNA ligase I, ATP dependent (Lig1)

Minichromosome maintenance deficient 7 (Mcm7)
Microarray

Fold Change

$+1.0$

$+1.0$

$-1.2$

$+1.1$

$+1.4$

$+1.7$

$+1.2$

$-1.1$

$-1.5$

$+1.5$

$+1.7$

\section{B. Transcription elongation}

Microarray

Gene Name

Fold Change

Elongin A (Tceb3)

$-4.8$

SII (Tcea1)

$-1.7$

ELL (EII)

$+1.1$

CA150 (Tcerg1)

$-1.2$

pTEFb

Cyclin-dependent kinase 9 (Cdk9)

$+1.0$

Cyclin T1

$-1.1$

Cyclin T2

$-1.3$

-Fold reductions (-) or increases (+) in Elongin A-/- MEFs of a subset of genes involved in DNA replication (A) or transcription elongation $(B)$ are listed. 